\title{
Acyclovir-Responsive Glioma: The Need for Vigilant Diagnosis
}

\author{
Young Gi Min, MD* (D); Haelim Kim, MD* (D); Seon Deuk Kim, MD* (D; Woo-Jin Lee, MD* (D); \\ Seung Geun Song, $\mathrm{MD}^{\dagger}$ (D); Sang Hyung Lee, $\mathrm{MD}, \mathrm{PhD}^{\ddagger} \mathbb{D}$; Yong-Seok Lee, $\mathrm{MD}, \mathrm{PhD}^{\mathbb{f}}$ (D) \\ Department of Neurology, Seoul National University Hospital ${ }^{\star}$, Seoul; Department of Pathology, Seoul National University Hospital ${ }^{\dagger}$, Seoul; \\ Department of Neurosurgery, Seoul Metropolitan Government-Seoul National University Boramae Medical Center, Seoul National University \\ College of Medicine ${ }^{\ddagger}$, Seoul; Department of Neurology, Seoul Metropolitan Government-Seoul National University Boramae Medical Center, \\ Seoul National University College of Medicine ${ }^{\mathfrak{l}}$, Seoul, Korea
}

We report a case of an acyclovir-responsive glioma, which was initially misdiagnosed as herpes simplex encephalitis (HSE). Intravenous acyclovir alone, without corticosteroid, dramatically improved the patient's symptoms and magnetic resonance imaging findings. He developed headache and amnesia after 1 year, when the stereotactic brain biopsy confirmed the final diagnosis of a glioblastoma, isocitrate dehydrogenase-1 wild-type. In retrospect, some of his sero-radiologic findings were not consistent with HSE. Polymerase chain reaction for herpes simplex virus in the cerebrospinal fluid tested negative. Parieto-occipital involvement and absence of post-treatment atrophy were also signs against HSE. We suggested that a low-grade glioma was present from the beginning and later underwent malignant transformation. The diagnosis of HSE should be made carefully. Acyclovir might also work on gliomas; this is confirmed by a typical clinical picture of HSE and a clear acyclovir-response. A vigilant review of the paraclinical features may shed light on the correct diagnosis.

J Neurosonol Neuroimag 2020;12(2):\$3-\$6

Key Words: Encephalitis, herpes simplex; Glioblastoma; Neuroimaging; Neuropathology
Received: June 9, 2020

Revised: June 30, 2020

Accepted: July 29, 2020

Correspondence:

Yong Seok Lee, MD, PhD

Department of Neurology, Seoul Metropolitan Government-Seoul National University Boramae Medical Center, Seoul National University College of Medicine, 20 Boramae-ro 5-gil, Dongjak-gu, Seoul o7o61, Korea

Tel: $+82-2-870-2473$

Fax: +82-2-831-0714

E-mail: mercades@snu.ac.kr
Glioblastoma (GBM) is a malignant tumor with a median survival of only 10-18 months even with standard treatment. ${ }^{1}$ We recently encountered a patient with GBM who experienced a transient "remission" on acyclovir treatment.

\section{CASE}

A 67-year-old man with no past medical illness presented with fever and myalgia, followed by a single brief episode of focal to bilateral tonic-clonic seizure. The neurological exam revealed no focal neurological deficit apart from anterograde amnesia noted from the Mini-Mental State Examination (28/30). Electroencephalogram revealed a moderate amount of generalized slowing with no epileptiform discharge. On the fluid-attenuated inversion recovery (FLAIR) images, homogenous high signal intensities were observed mainly in the left medial temporal lobe (Fig. 1A). The lesions were partially accompanied by diffusion restriction and extended to the left parieto-occipital lobe (Fig. 1B, C). Contrast enhancement was faintly present on the adjacent leptomeninges (Fig. 1D). Cerebrospinal fluid (CSF) exam revealed 0 white blood cells $/ \mu \mathrm{L}$, protein $71.6 \mathrm{mg} / \mathrm{dL}$, and glucose $74 \mathrm{mg} / \mathrm{dL}$. Polymerase chain reaction (PCR) was negative for herpes simplex virus (HSV) 1, HSV 2, varicella zoster virus, Mycobacterium Tuberculosis, and cytomegalovirus (CMV) on two consecutive CSF samples obtained 6 days apart.

Although the clinical manifestation closely resembled that of HSE, negative CSF HSV PCR led to another possibility, a glioma. However, the lesions were mostly confined to the medial temporal lobe and were too dangerous to biopsy. 

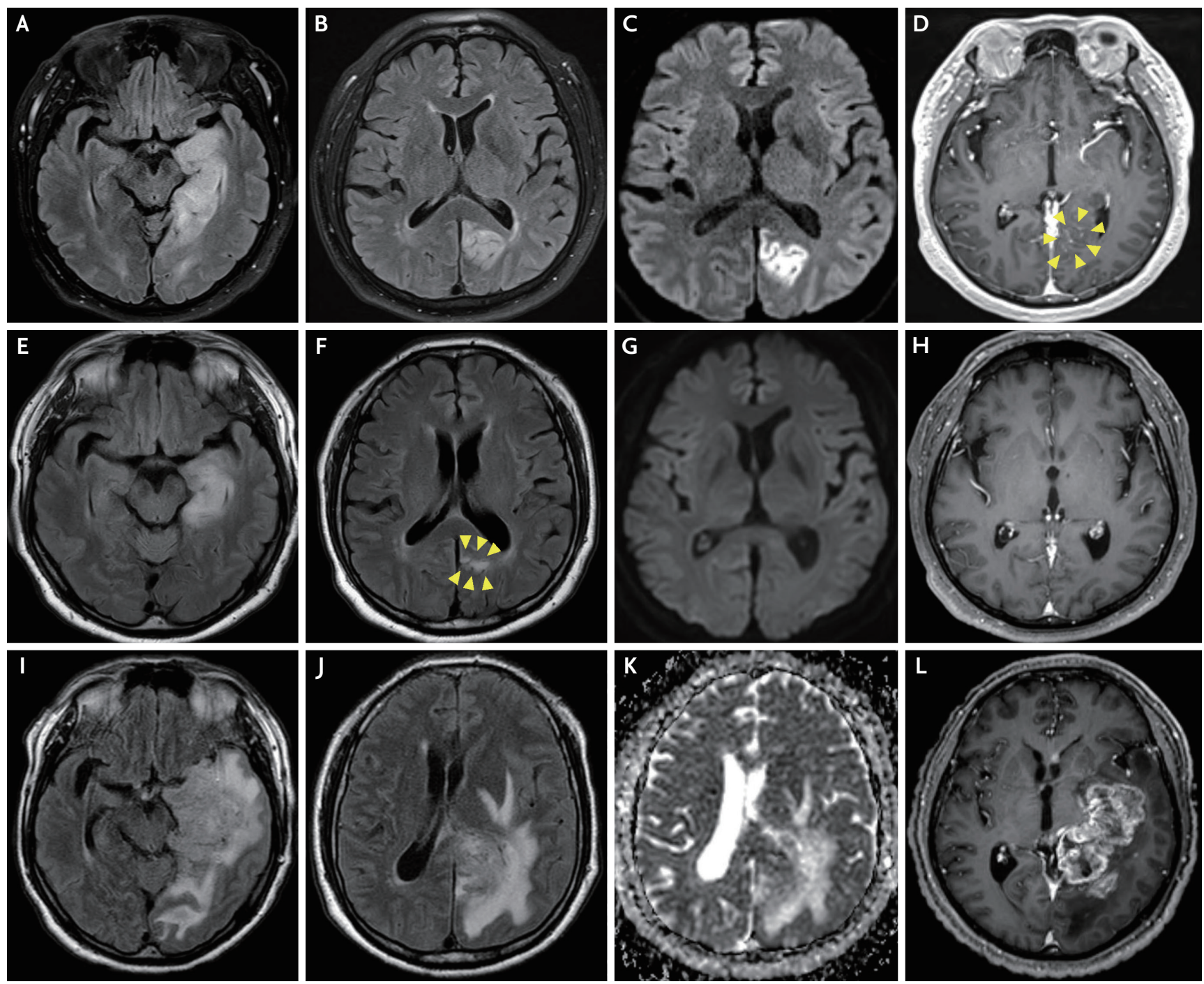

FIG. 1. Serial magnetic resonance imaging (MRI) changes (A-D) at the onset, $(E-H)$ immediate post-treatment, and (I-L) I year after treatment. (A) Fluid-attenuated inversion recovery (FLAIR) hyperintensities were noted mainly in the left medial temporal lobe. (B) The FLAIR lesions extended to the high parieto-occipital area and (C) were accompanied by diffusion restrictions (apparent diffusion coefficient [ADC] images not shown).

(D) Faint enhancement (marked in arrows) was noted in the adjacent leptomeninges. (E, F) Follow-up MRI 3 months after the treatment showed a remarkable decrease in the FLAIR lesions (marked in arrows). Focal cerebromalacic changes had developed in the high parietal cortex. (G) The previously noted diffusion restrictions and $(\mathrm{H})$ contrast enhancement were absent on the follow-up MRI. (I, J) MRI taken I year after the onset revealed extensive FLAIR lesions with a marked swelling and mass effect. (K) High ADC values with relatively low biooo (not shown) values indicate a predominant vasogenic edema in the peri-tumoral tissue. (L) Extensive parenchymal enhancement was noted.
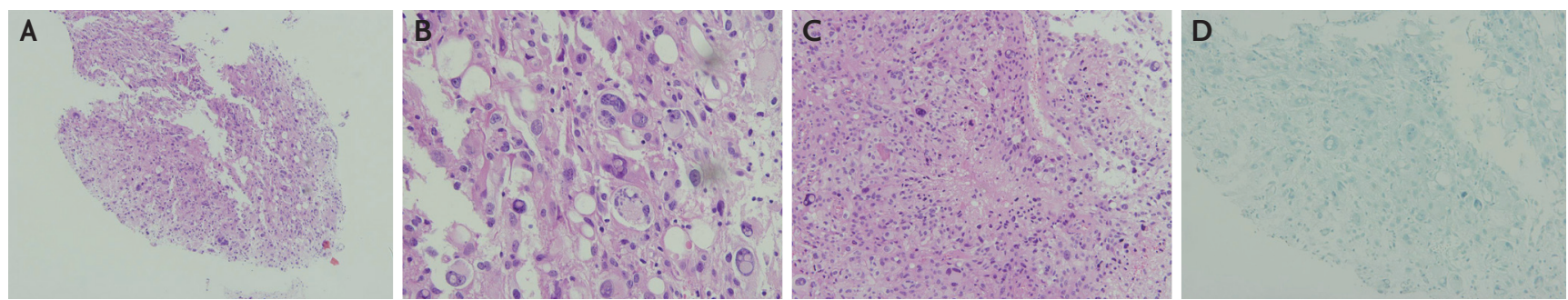

FIG. 2. Histopathological findings of the brain specimen from the stereotactic biopsy. (A) Fragmented brain tissue showing hypercellular neoplastic lesions with no normal brain parenchyma ( $\mathrm{H} \& \mathrm{E}, \times 100)$. (B) At a higher magnification, bizarre-looking giant cells with a marked nuclear pleomorphism were noted (H\&E, $\times 400)$. (C) Necrotic zone surrounded by the atypical tumor cells forming a pseudopalisading pattern (H\&E, $\times 200)$. (D) Immunohistochemical staining was negative for herpes simplex virus $(\times 200)$. 
Moreover, his symptoms were too minor. Therefore, the patient was first started on intravenous acyclovir treatment for 2 weeks, which surprisingly improved his symptoms and magnetic resonance imaging (MRI). MRI obtained 3 months after the treatment revealed markedly reduced FLAIR lesions, diffusion restriction, and leptomeningeal enhancement (Fig. 1E-H). No new focus of disease was noted. Corticosteroids had not been administered.

He visited Boramae Medical Center 1 year later for headaches and memory loss that had developed 2 weeks prior. Recurrent episodes of nonconvulsive seizures were suspected. MRI showed extensive heterogenous FLAIR hyperintensities accompanied by a mass effect (Fig. 1I). Contrast enhancement showed the breakdown of blood-brain barrier (Fig. 1J). High values on apparent diffusion coefficient suggested a vasogenic edema (Fig. 1K). In addition, parenchymal hemorrhage was newly noted on the susceptibility-weighted image (Fig. 1L). Stereotactic biopsy performed from the lateral temporal lobe revealed anaplastic cells with microvascular proliferation and necrosis (Fig. 2A-C). On immunohistochemistry (IHC), positive glial fibrillary acidic protein and negative isocitrate dehydrogenase-1 (IDH-1) confirmed the diagnosis of a glioblastoma, IDH-1 wild-type. IHC for p53 was negative and for ATP-dependent helicase (ATRX) was positive (no mutation). Nuclear viral cytopathic changes indicating HSV infection were not observed. In addition, IHC using antibodies to HSV1, HSV2, and CMV antigens were negative (Fig. 2D).

\section{DISCUSSION}

Several clues suggest that temporal lobe lesions were not HSE but a glioma from the beginning. First, two consecutive CSF HSV PCR tests, the widely accepted test of choice for HSE with $98 \%$ sensitivity, were negative. ${ }^{2}$ Second, initial MRI lesions outside the limbic regions are associated with lower odds of HSE. ${ }^{3}$ Third, the absence of brain atrophy following antiviral treatment is rare in HSE. ${ }^{4}$ Finally, there was no histopathological evidence of HSE in the biopsied brain. This raised a low possibility of coincident HSE. Altogether, it was assumed that a glioma was transiently suppressed by acyclovir. Based on the MRI features and the 1-year latency before aggravation, its grade would have been II or III, and later progressed to glioblastoma multi- forme.

Theoretically, acyclovir could suppress regulatory $\mathrm{T}$ cell function and therefore amplify the immune reaction toward the tumor tissue. ${ }^{5,6}$ However, clinical experience is largely limited to case reports, in which GBM patients were misdiagnosed and treated as HSE. ${ }^{7-9}$ Moreover, some patients among them had received corticosteroids concomitantly; this made it difficult to judge the effect of acyclovir. ${ }^{9}$ In addition, HSE occasionally coincides with gliomas; in these cases, acyclovir would act by reducing the viral load but not the size of the glioma. ${ }^{10}$ In contrast, we suggest a clear-cut example in which we administered acyclovir without corticosteroid and objectified the improvement on a follow-up MRI.

Physicians should be cautious when diagnosing HSE, a typical clinical picture and a clear response to acyclovir does not guarantee the diagnosis. Paraclinical features should be reviewed carefully. As long as those findings are atypical for HSE, physicians should consider a brain biopsy. If it is not feasible, a long-term surveillance with frequent MRI check-ups is recommended.

\section{Conflicts of Interest}

No potential conflicts of interest relevant to this article was reported.

\section{REFERENCES}

1. Hanif F, Muzaffar K, Perveen K, Malhi SM, Simjee SU. Glioblastoma multiforme: a review of its epidemiology and pathogenesis through clinical presentation and treatment. Asian Pac J Cancer Prev. 2017;18:3-9.

2. Galen BT. Screening cerebrospinal fluid prior to herpes simplex virus PCR testing might miss cases of herpes simplex encephalitis. J Clin Microbiol. 2017;55:3142-3143.

3. Chow FC, Glaser CA, Sheriff H, Xia D, Messenger S, Whitley $\mathrm{R}$, et al. Use of clinical and neuroimaging characteristics to distinguish temporal lobe herpes simplex encephalitis from its mimics. Clin Infect Dis. 2015;60:1377-1383.

4. Soto-Hernandez JL. Follow-up in herpes simplex virus encephalitis. Clin Infect Dis. 2000;31:206-207.

5. Kominsky SL, Subramaniam PS, Johnson HM, Torres BA. Inhibitory effects of IFN-gamma and acyclovir on the glioblastoma cell cycle. J Interferon Cytokine Res. 2000;20:463-469.

6. Söderlund J, Erhardt S, Kast RE. Acyclovir inhibition of 
IDO to decrease Tregs as a glioblastoma treatment adjunct. J Neuroinflammation. 2010;7:44.

7. Piper K, Foster H, Gabel B, Nabors B, Cobbs C. Glioblastoma mimicking viral encephalitis responds to acyclovir: a case series and literature review. Front Oncol. 2019;9:8.

8. Smithson E, Larner AJ. Glioblastoma multiforme masquerading as herpes simplex encephalitis. Br J Hosp Med (Lond). 2013;74:52-53.
9. Nam TS, Choi KH, Kim MK, Cho KH. Glioblastoma mimicking herpes simplex encephalitis. J Korean Neurosurg Soc. 2011;50:119-122.

10. Berzero G, Di Stefano AL, Dehais C, Sanson M, Gaviani P, Silvani A, et al. Herpes simplex encephalitis in glioma patients: a challenging diagnosis. J Neurol Neurosurg Psychiatry. 2015;86:374-377. 